Genet. Res., Camb. (1964), 5, pp. 282-304

With 5 text-figures

Printed in Great Britain

\title{
A mechanism for gene conversion in fungi
}

\author{
BY ROBIN HOLLIDAY \\ John Innes Institute, Bayfordbury, Hertford, Herts.
}

(Received 17 January 1964)

In many fungi it is possible to examine directly the four haploid meiotic products. Such tetrad analysis has demonstrated that any heterozygous locus normally gives the expected 2:2 Mendelian ratio; but that aberrant tetrads also occasionally occur, where one allele is represented three times and the other once. This aberrant segregation was first clearly demonstrated in yeast by Lindegren (1953) and is usually referred to as gene conversion. In the last ten years gene conversion has been demonstrated in a number of fungi both at meiosis and mitosis, and recent reviews of much of this work are available (Roman, 1963; Fincham \& Day, 1963). Many examples of gene conversion have been discovered in heteroallelic crosses, that is, in crosses between different mutant alleles of the same gene. Such crosses can generate rare wild-type progeny if one of the mutant alleles segregates abnormally, so that only a single copy of the mutant allele is present in a tetrad. This type of gene conversion was first detected by Mitchell (1955) in Neurospora and has often been referred to as non-reciprocal recombination. In this paper this term will be used synonymously with gene conversion. In general, tetrad analysis of crosses between allelic mutants shows either non-reciprocal recombination (conversion for one or the other allele), or a mixture of this with reciprocal recombination, where both alleles show a 2:2 segregation, but a cross-over or reciprocal exchange between the alleles results in the production of a wild-type allele and a doubly mutant one. Gene conversion may also occur in higher organisms such as maize (Nelson, 1962) or Drosophila (Hexter, 1963), although in the absence of tetrad analysis it is not usually possible to distinguish with certainty between conversion and clusters of reciprocal exchanges. Such clusters, resulting from high negative interference operating over short regions of effective pairing, had been postulated from other work in fungi (Pritchard, 1955) where crosses between auxotrophic alleles had been analysed by selecting prototrophic recombinants and examining these for their genotype with respect to linked outside markers.

Gene conversion has been most frequently explained by supposing that genetic replication occurs during or after chromosome pairing; that the replicas can switch from copying off one chromosome to copying off the other in short regions of effective pairing, and that the switching need not be reciprocal. In order for normal chromatid formation to occur on each side of this region the sum of all switches must be an even number. This copy choice model can explain 3:1 ratios for particular alleles and it can also be used to explain classical crossing-over. There is in fact good evidence that 
crossing-over and conversion are correlated and tend to affect only two of the four chromatids (Perkins, 1962; Whitehouse, 1963). A number of models based on the same basic copy choice hypothesis have been considered, and a recent detailed discussion of some of these is available (Stadler \& Towe, 1963). One advantage of the copy choice hypothesis is that it makes several quite specific predictions: (1) that the genetic material replicates conservatively; (2) that genetic pairing must take place prior to or at the same time as genetic replication; (3) that if the hypothesis is used to explain crossing-over as well as conversion, and sister strand exchange by breakage and reunion is ruled out, then successive cross-overs along the length of the chromosome should involve the same two chromatids; (4) that at a given heterozygous site conversion from mutant to wild allele and from wild to mutant allele should occur with equal frequency, and (5) that in crosses between different mutants within the same gene, the frequency of conversion to the wildtype allele should be proportional to the distance between the mutants, thus allowing the construction of linear maps with additive recombination frequencies.

It has been evident for some time that predictions (1), (2) and (3) are not supported by experimental evidence. These difficulties of the copy choice model have been thoroughly discussed elsewhere (Pritchard, 1960 a; Fincham \& Day, 1963; Whitehouse, 1963). In addition there is accumulating evidence that prediction (4) is not borne out either. Kitani, Olive \& El-Ani (1962) found that in crosses between a spore colour mutant and wild-type in Sordaria, conversion from mutant to wild allele occurred about one-fifth as frequently as conversion in the reverse direction. Rizet \& Rossignol (1964) recently reported similar asymmetries in Ascobolus; and data from two sources (see below) would appear to demonstrate the same effect in Neurospora. On the other hand, prediction (5) appears to be well substantiated in a number of studies.

In spite of its difficulties the copy choice hypothesis is still favoured by a number of authors (Stadler \& Towe, 1964; Kitani et al., 1962; Lissouba, Mousseau, Rizet \& Rossignol, 1962). This is so because it is assumed that conversion is impossible without some genetic replication; that since conversion frequencies can be used to construct linear gene maps it is assumed that conversion must be due to a recombinational event, and, finally, because the striking polarized conversion discovered in Ascobolus (Lissouba et al., 1962) appears to be reasonably explicable only if polarized replication and switching occurs. None of these authors has suggested a mechanism which would allow very precise accuracy in the miscopying process. Genetic results can only be explained if the switch takes place in such a way that no deletion or duplication of the genetic material occurs. It is simply accepted that given close genetic pairing (by another unspecified mechanism), accurate switching could operate. This being so, it is rather surprising that recombination by breakage and reunion has often been disregarded on the grounds that it could not be a process precise enough to explain the genetic data. It is very relevant to this discussion that in bacteria and bacteriophage, where recombination data had for some time been explained on the basis of a copy choice model, there is now good evidence for recombination by breakage and reunion of parental genetic material (Meselson \& Weigle, 
1961 ; Kellenburger, Zichichi \& Weigle, 1961 ; Siddiqi, 1963), and even more significant, evidence for genetic recombination in the absence of deoxyribonucleic acid (DNA) synthesis (J. J. Weigle, personal communication).

In this paper an origin for conversion events which does not involve genetic replication will be discussed. Although the mechanism proposed at first sight explains the raw data much less readily than does copy choice, it avoids the difficulties this theory encounters, and it makes predictions some of which are confirmed by observations already recorded in the literature, and others which could be tested experimentally.

\section{THE MODEL}

The model to be described has already been discussed briefly in an earlier publication (Holliday, 1962), and Whitehouse (1963) has recently put forward a detailed mechanism for crossing-over based on the same principle; although in his scheme some genetic replication is required. In order to simplify the description of the model it will be assumed that the genetic material in the chromosome consists of a single DNA double helix. The model is not basically incompatible with a polyneme or multistranded chromosome, but full consideration of this possibility would make it necessary to introduce further assumptions that might serve only to confuse the general argument. For the same reason it will be assumed that mutations involve only a single base pair substitution. (It has in fact been shown by very extensive studies in Schizosaccharomyces that virtually all induced mutations are point mutations rather than small structural changes (Leupold, 1961; Gutz, 1961).) The products of replication will be referred to as chromatids, although of course cytologically visible chromatids appear only some time after genetic replication at meiosis. Two levels of genetic pairing will be assumed. General genetic pairing that brings the chromatids into close contact along their length, and specific or effective pairing (Pritchard, 1955) which allows molecular contact over short regions of the genetic material within which conversion and crossing-over can occur.

This model uses the complementarity of the two strands of DNA in order to explain specific chromatid pairing at the molecular level. After genetic replication and general genetic pairing, at certain points DNA molecules from opposite homologous chromatids unravel to form single strands, and these then anneal or coil up with the complementary strands from the other chromatid. Thus specific or effective pairing over short regions could occur. There are a number of possibilities of varying complexity as to how the DNA might get into this configuration; but since at this time it would seem desirable to present the general features of the model rather than discuss detailed alternatives, only one of the simplest possibilities will be described. This is shown in diagrammatic form in Fig. 1.

It is supposed that strand separation begins at a defined point, perhaps a linker between contiguous DNA molecules. Genetic evidence for such discontinuities is already available (Lissouba et al., 1962; Murray, 1963). Strand separation proceeds along the length of the two molecules, but can end at any point. Annealing of strands then occurs followed by re-attachment of the free ends to the linker. 
The chromatids are now attached at an arbitrary point by a chiasma-like structure involving single strands (half chromatids) of DNA. It is postulated that at the points where strands exchange partner precise breakage and reunion of non-complementary strands can occur so that there is no deletion or duplication in either strand.

If one of the pairs of non-complementary strands is involved in this process, then the half chromatid chiasma is converted into a whole chromatid chiasma, thus

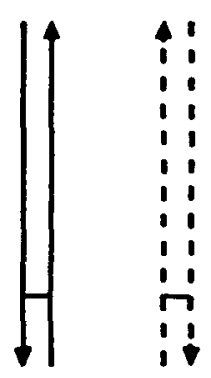

1

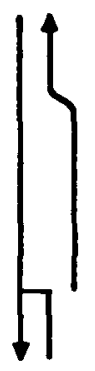

2

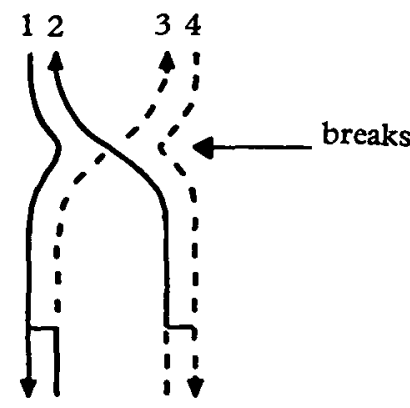

3

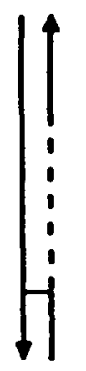

$4 a$

(strands 2 and 3 break and rejoin) hybrid region within which conversion can occur

Fig. 1. Diagramatic illustration of the successive stages of effective pairing and recombination of homologous chromatids. Solid lines represent the DNA strands of one chromatid and broken lines those of the other chromatid. The polarity of the strands is indicated by the arrows, and the short horizontal line gives the position of the linker or genetic discontinuity.

recombining outside markers and permitting the mechanical separation of chromatids which must occur at anaphase (Fig. 1, 4b). If the other pair is involved then no chiasma is formed and there is no recombination of outside markers (Fig. 1, 4a). In both cases there is a region of 'hybrid' DNA extending from the linker to the point of breakage. If this part of the genetic material is homozygous then normal base pairing will occur in the hybrid region; but if the annealed region spans a point of heterozygosity - a mutant site-then mispairing of bases will occur at this site. It is further postulated that this condition of mispaired bases is unstable. It has been shown that bases which are not held within the double helix by normal 
hydrogen bonding, can swing out from and return within the helix without disturbing its structure (Fresco \& Alberts, 1960). One or both such bases may get involved in exchange reactions with free bases, so that when a complementary pair is present these could swing back and be retained within the helix by normal hydrogen bonding. It is most reasonable to suppose that such exchange reactions would be enzyme mediated. There is a rather obvious connection between this suggestion and the growing evidence for mechanisms in the cell which can repair DNA damaged by mutagens (see Sobels, 1963). If there are enzymes which can repair points of damage in DNA, it would seem possible that the same enzymes could recognize the abnormality of base pairing, and by exchange reactions rectify this. Regions of hybrid DNA would thus provide the opportunity for mutation like changes in genes which are heterozygous. Depending on which direction these changes occurred, $1: 3$ or 3:1 ratios for particular alleles could be achieved in the absence of any DNA synthesis. If the change did not occur, or occurred in only one of the two pieces of hybrid DNA, before the next replication after meiosis, then post meiotic segregation would occur such as has sometimes been observed in Sordaria (Kitani et al., 1962), Ascobolus (Lissouba et al., 1962) and Neurospora (Stadler \& Towe 1963).

\section{IMPLICATIONS OF THE MODEL}

The proposed mechanisms for conversion and crossing-over is free from the difficulties the copy choice model encounters. Conversion and crossing-over can take place after genetic replication and at the time when chromatids are visibly paired. Two homologous chromatids are involved in the short region of effective pairing, but such regions at different positions along the chromosome may involve different pairs of chromatids. Thus three-strand and four-strand as well as twostrand cross-overs are expected on the basis of this model, while they require special explanation on any copy choice hypothesis. The mechanism could also explain why for a given heterozygous site the conversion rate from mutant to wild may differ from that in the reverse direction. Of the twelve possible types of mispairing, some may be corrected more easily than others; or, the correction may not be random but directed in a specific manner. This would also explain why some mutants do not show post meiotic segregation whilst others do so (Lissouba et al., 1962; Kitani et al., 1961).

Along with copy choice, the model explains why conversion and crossing-over are correlated, why when both events do occur only two of the four chromatids are involved, and why conversion can occur without crossing-over. In crosses between different mutant alleles non-reciprocal or reciprocal intragenic recombination could be observed depending whether the hybrid region spans one or both mutant sites, and in which ways the mispairings were corrected. Mutants which were on either side of the linker would tend to show reciprocal recombination, since the region of hybrid DNA would often lie between them. These points will be discussed in more detail below, in relation to specific data.

On the other hand, the model does not appear to explain why conversion rates in 
crosses between mutants of the same gene should vary according to their distance apart. In other words the model does not appear to be reconcilable with the fact that linear gene maps can be constructed on the basis of conversion frequencies. Any mutant within a region of effective pairing should have the same opportunity to undergo conversion irrespective of its distance from another mutant in the same gene. Crosses between closely linked mutants could give wild-type progeny with a frequency comparable to that from crosses between widely spaced mutants.

This would not be so if the mutant sites themselves inhibited genetic pairing. This suggestion of course contradicts the long and widely held belief that the presence of genetic markers does not interfere with recombination and chromosome mapping. But although it goes against this part of accepted theory, it is almost what one might predict from another aspect of accepted genetic theory. This is that genetic pairing requires genetic homology; as homology decreases genetic pairing is reduced or prevented. A few sites of heterozygosity in otherwise homologous chromosomes may not upset general pairing, but may influence the location of the regions of effective pairing. In standard genetic crosses where long regions of homozygous genetic material occur between genetic markers, these markers would have no apparent effect on crossing-over and mapping, but when recombination in the immediate vicinity of markers is being examined, an effect of heterozygosity may operate. Two sites close together may interfere more strongly with genetic pairing than those further apart. Since we know nothing about the forces that operate in general genetic pairing it is fruitless to speculate as to how the presumed inhibition could act at this level. However, at the level of effective pairing points of heterozygosity would clearly tend to disturb or terminate the annealing process, since this depends on genetic homology, and the chance of annealing failing may vary according to the distances apart of the mutant sites. (It should be pointed out that the idea that genetic markers may affect recombination frequencies is not a new one: but previous discussions along these lines have argued that markers may stimulate rather than inhibit recombination (Hershey, 1958; Pontecorvo, 1958).)

If there is an inhibiting effect by mutant sites on the opportunity for pairing, conversion and crossing-over, then the degree of inhibition might be inversely proportional to the distance apart of such sites. In this case gene maps with additive recombination frequencies would result. However, strict additivity would only obtain if all the mutants inhibited pairing to the same extent, and if all mutants showed the same conversion frequency when within the hybrid region. These conditions are unlikely to be rigorously fulfilled, so what might be expected is the kind of tantalizing result that is often found: a general linearity of mutant sites, with departures from additive recombination frequencies significant enough to make the precise order of sites ambiguous. Over short distances, however, it would not be surprising if recombination frequencies increased in proportion to more than the first power of the distance separating sites: the intensity of inhibition might, for example, obey something approaching an inverse square law. In these circumstances the tightness of linkage of relatively close sites would appear exaggerated in gene maps. In other words mutant sites which were close together on the DNA of the 
gene would appear correspondingly closer on the map; the frequency of wild-type progeny they generate when crossed would not be a real measure of their distance apart, but some value less than this. This would result in linear gene maps without strict additivity of recombination fractions owing to the occurrence of map expansion. In a gene map the length of the intervals between adjacent mutant sites is given by the frequency of recombinants these mutants generate when intercrossed, but with map expansion the frequency of recombinant progeny from crosses between mutants several intervals apart will be greater than the sum of all the inter vening intervals. Up to now map expansion has not been recognized, but an examination of published data points very strongly to its being a real effect. It has no explanation in current theory, indeed it conflicts with normal mapping principles. In the three-point cross it is quantitatively impossible for the recombination of outside markers to exceed the sum of the recombination between adjacent markers; hence expansion in maps based on two-point crosses, must mean that the mutant sites are themselves interfering with the process of conversion and crossingover. In the three-point intragenic cross recombination would be additive, but the outermost markers would recombine less frequently than when the centre marker is absent from the cross. The only departure from additivity that has hitherto been expected has been in the opposite direction to map expansion, with more distant sites showing closer linkage in the two-point cross than the sum of the intervening intervals would suggest. This is what would be predicted if negative interference was operating in the given region, but in fine structure maps in fungi this type of departure from additivity is not observed.

A corollary of map expansion would be the tendency for mutants to be distributed in clusters separated by empty regions in the fine structure map. Such a tendency is clearly demonstrated in most gene maps in fungi. Clustering has previously been explained (e.g. Siddiqi, 1962) as being due either to areas in the gene which are relatively non-mutable, or, alternatively, that mutations in certain parts of the gene have little or no phenotypic effect, and therefore would not be isolated. Although these explanations seem plausible for occasional examples of clustering, it would be surprising if almost every gene showed such discontinuity in mutability or phenotypic effect.

Clearly the mechanism of conversion and crossing-over that is proposed is a general rather than a specific one, since it contains many features which are difficult to treat in any quantitative way. These features are: the manner in which mutants may affect both general pairing and annealing; the length of the annealed region in relation to map distance and the variation of this length; the probabilities of repair of mispaired bases and whether this repair is random or follows distinct patterns, and, finally, whether the two pairs of non-complementary strands break and rejoin with equal probability. For these reasons it would not be possible to formulate a mathematical model other than one which would further increase the speculation by imposing additional assumptions. Moreover, there are strong indications that whatever basic mechanism is operating, the details of this mechanism may not be the same in different organisms; therefore it does not seem profitable at the present time 
to attempt to make the model more specific by very detailed analysis of particular data from one organism or one locus. Instead, in the ensuing discussion pertinent information from each organism in turn will be briefly examined in relation to what has been previously discussed: namely, the clustering of sites; non-additivity of recombination fractions and map expansion; non-linearity of sites; post-meiotic segregation; asymmetric and polarized conversion, and evidence for discontinuities in the genetic material.

\section{DATA FROM TETRAD AND FINE STRUCTURE ANALYSIS}

(i) Schizosaccharomyces pombe

By far the most extensive fine structure mapping in fungi has been carried out with this yeast. Very detailed maps of the $a d_{7}$ (Leupold, 1961) and $a d_{6}$ (Leupold \& Gutz, personal communication) genes have been constructed. These are based on prototroph frequencies from crosses between mutants at these two loci. Tetrad analysis of crosses between alleles have shown that intragenic recombination is predominantly non-reciprocal (Leupold, personal communication). The $a d_{7}$ locus consists of thirty-three sites, but only fifteen of these appear in the map where recombination frequencies are given. Eight of these form a distinct cluster near one end of the map, and at the other end of the map there is a large region free of sites. The map of the $a d_{6}$ region consists of thirty-three mutant sites, and over 200 crosses between pairs of these have been made. Again, the sites do not appear to have a random distribution. In both maps the order of mutant sites is for the most part unambiguous. However, although linearity is convincing, the recombination frequencies are not additive, since the phenomenon of map expansion is strikingly demonstrated. If map distance is expressed as the sum of all the shortest intervals between two sites which are several intervals apart, then this distance is appreciably less than the recombination frequency when these two mutants are themselves crossed. These results are shown for the two loci in Figs. 2 and 3. The expansion effect is not apparent when short regions of the gene maps are examined and these data are omitted from the graphs. It should be pointed out that if some of the mutants were sizeable deletions, a map expansion effect might result; but in fact the data clearly show that none of the mutants are deletions of this type. The data can only be reasonably interpreted as evidence for the inhibition by mutant sites of the processes of intragenic recombination.

\section{(ii) Aspergillus nidulans}

In fine structure studies at the ad8 locus the analysis of half tetrads following intragenic recombination in heteroallelic diploids showed that such recombination could be reciprocal (Pritchard, 1955, 1960b). More recent and extensive half tetrad analysis at the paba1 locus indicate that intragenic recombination may be reciprocal or non-reciprocal (Putrament, 1963). Fine structure mapping at these two loci has been carried out by selecting prototrophs from crosses between mutant alleles and then classifying these with respect to outside markers. The most detailed 


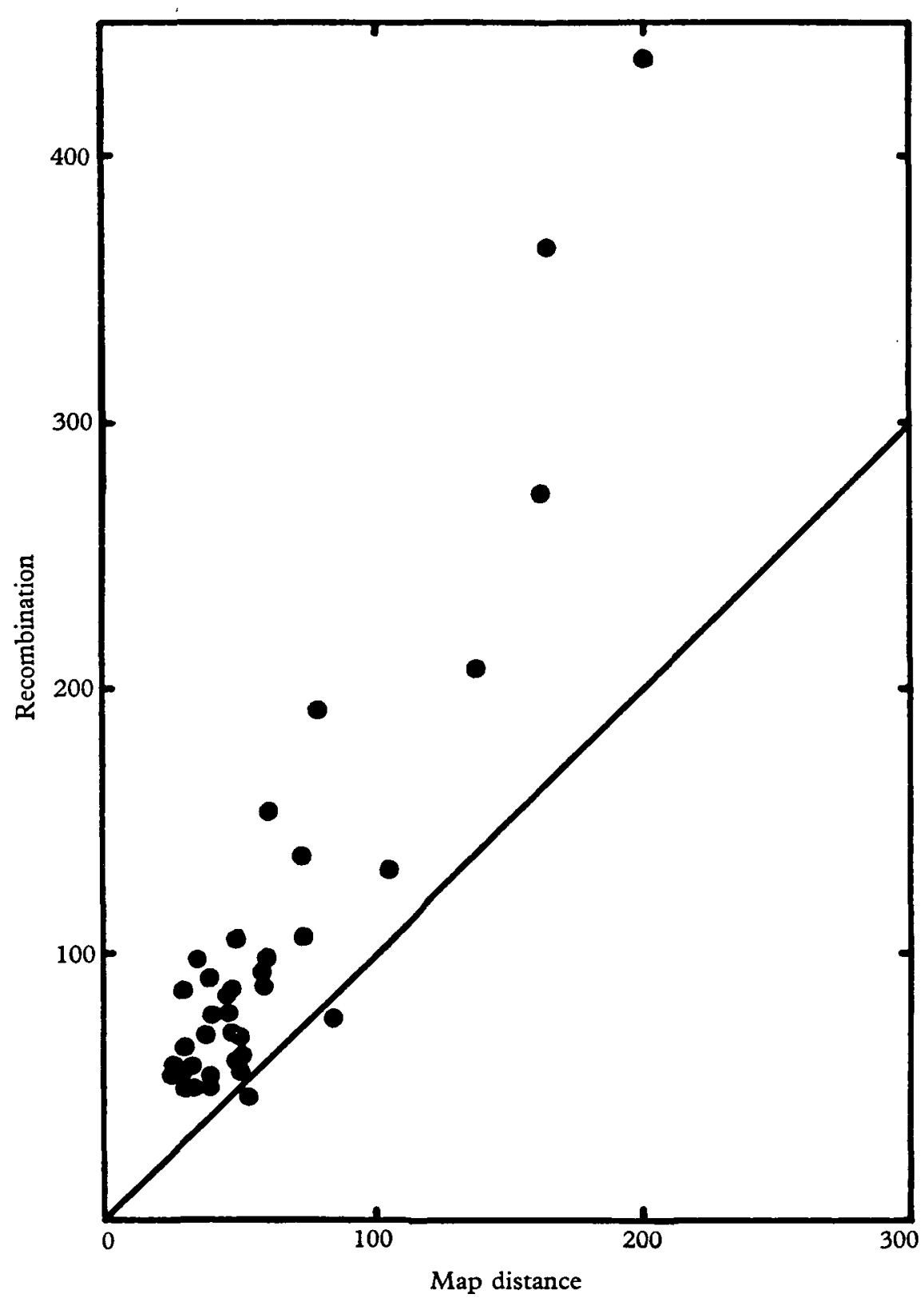

Fig. 2. Recombination as a function of map distance for Schizosaccharomyces $a d_{8}$ mutants. For each point the ordinate is the measured frequency of recombination for a given pair of mutants expressed as prototrophs per million ascospores; and the abscissa is the sum of the recombination values for the smallest intervals between the given pair of markers. Points are only included where either the map distance or the recombination frequency is greater than 50 per million, and where the map distance is the sum of at least six intervals. 


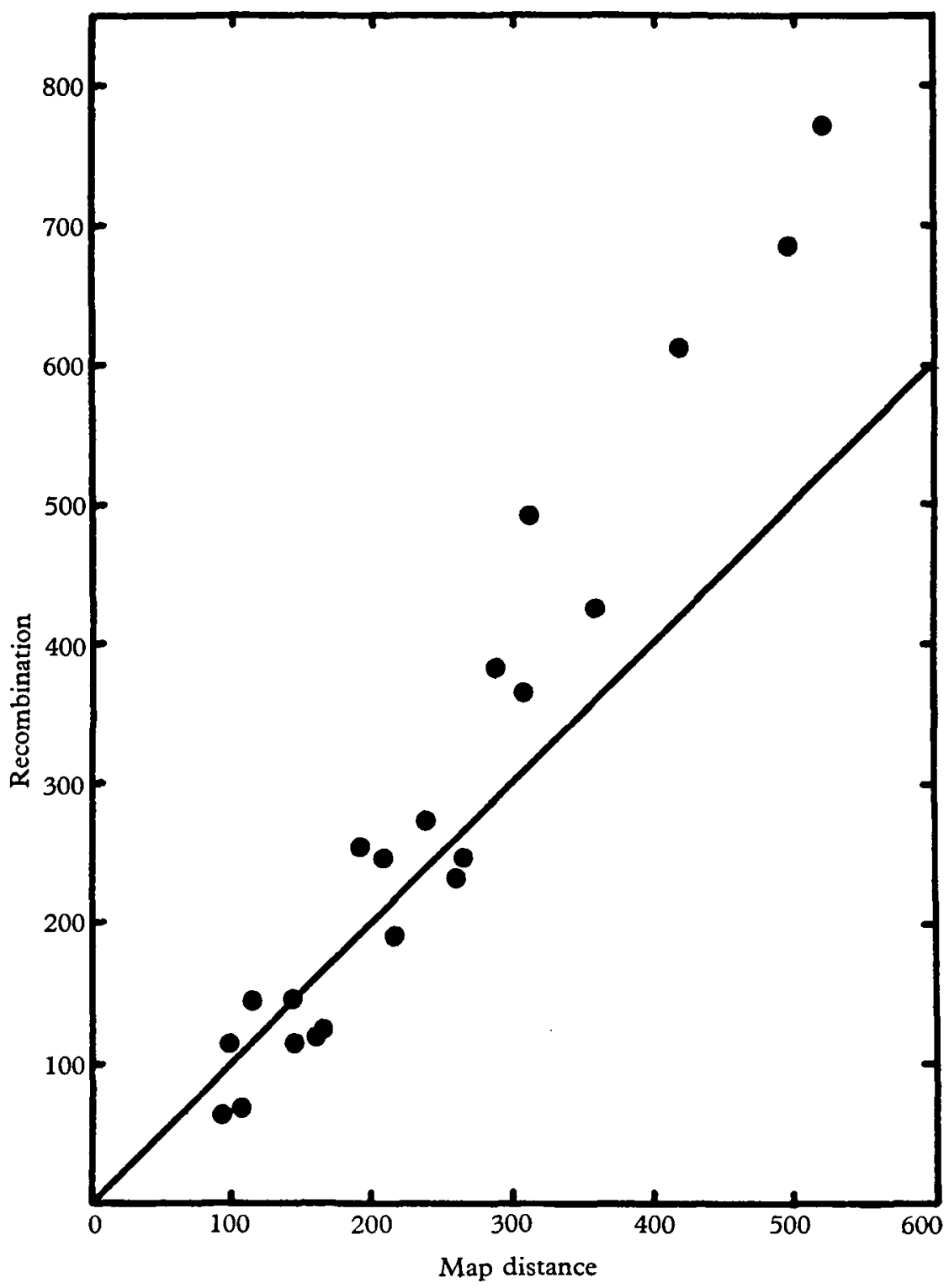

Fig. 3. Recombination as a function of map distance for Schizosaccharomyces ad, mutants. For each point the ordinate is the measured frequency of recombination for a given pair of mutants expressed as prototrophs per million ascospores; and the abscissa is the sum of the recombination values for the smallest intervals between the given pair of markers.

map is that of the paba1 region (Siddiqi, 1962). The map shows extreme clustering: ten sites are all at the proximal end and occupy approximately one-twentieth of the total map length, and the rest of the map consists of only two widely spaced sites. The convincing linearity of sites is based both on prototroph frequencies and the behaviour of outside markers. However, the recombination frequencies are non- 
additive : the cluster region shows even more pronounced map expansion than do the data from Schizosaccharomyces, and the results are shown in Fig. 4. Again the expansion effect is not apparent when the recombination between sites only two or three intervals apart is compared to the map distance. In addition, the expansion is

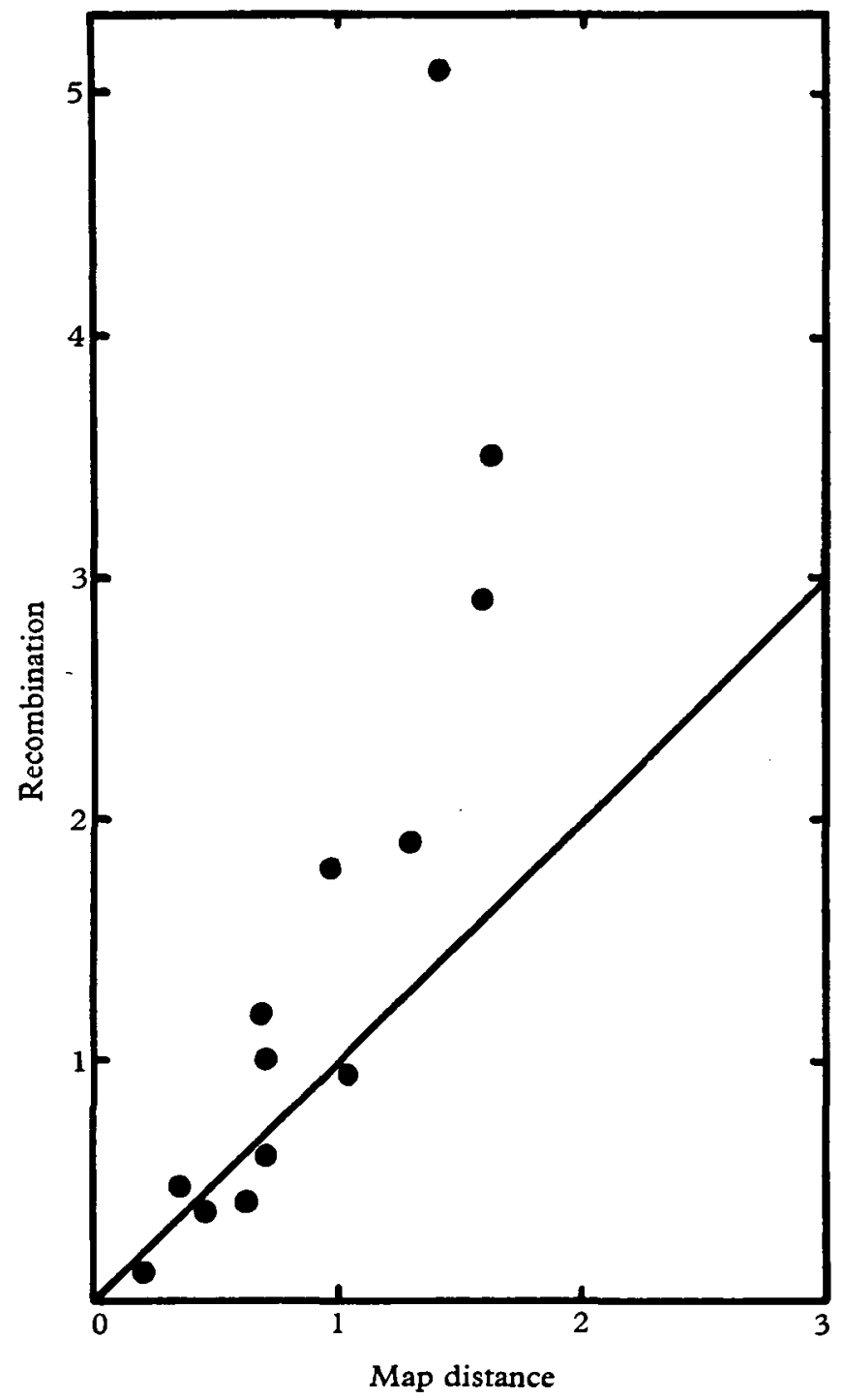

Fig. 4. Recombination as a function of map distance for Aspergillus paba1 mutants. For each point the ordinate is the measured frequency of recombination for a given pair of mutants $\times 10^{5}$; and the abscissa is the sum of the recombination values for the smallest intervals between the given pair of markers.

less pronounced though still apparent when the data from the two distal sites are examined. Owing to the disproportionate recombination frequencies these mutants give, these data have not been included in the graph. The map of the ad- 8 
locus (Pritchard, 1960b) is less detailed but it again shows an apparent random distribution of linearly arranged sites and a tendency towards map expansion. It is interesting to note that from the behavour of outside markers it has been supposed in both studies that negative interference is operating over short intervals, whereas in fact such negative interference would be expected to result in the reverse of a map expansion effect.

According to the negative interference model, in both these studies an exchange between the mutant alleles is accompanied frequently by an additional exchange between the distal allele in the cross and the distal outside marker, and much less frequently by an exchange between the proximal allele and the proximal marker. This polarity in outside marker recombination disappears when the alleles in a cross are very tightly linked (Siddiqi \& Putrament, 1963). This effect could be consistent with the proposed mechanism for gene conversion if a discontinuity in the genetic material exits between the most distal mutant site and the distal marker. If annealing extended proximally from this discontinuity and tended to include only the distal mutant in the cross, then conversion could occur at this site. Conversion with crossing-over between outside markers (see Fig. 1, 4b) would give the same result as a supposed reciprocal exchange between the mutant sites; and conversion without outside marker recombination (Fig. 1, 4a) would give the same result as a supposed reciprocal exchange between the alleles, along with one distal to them. The other two outside marker configurations are observed much more rarely and could arise if the annealing spanned both mutant sites. In crosses between very tightly linked sites where the polarized effect disappears, it could be supposed that the annealed region rarely reaches the mutants, but when it does so, both sites tend to be included.

\section{(iii) Neurospora crassa}

Gene conversion has been demonstrated by tetrad analysis of interalleleic crosses at four separate loci. The most extensive studies have been by Case \& Giles (1958, 1963 ) with the pan-2 locus and by Stadler \& Towe (1963) with the cys locus. The fine structure map of the pan-2 region is based on random spore prototroph frequencies. The map shows a distinct cluster of sites at the proximal end, and the general linear order of sites is by no means convincing. Since the order of sites is ambiguous and the number of crosses which have been performed is not large, it is not possible to determine whether map expansion is present or not. The extensive tetrad analysis shows that intragenic recombination can be by conversion or, less commonly, by reciprocal recombination. The data have been explained by the copy choice hypothesis, but three features do not seem to be easily compatible with it. (1) One of the mutants used, mutant 5 , showed conversion from mutant to wild on eleven occasions and no conversion in the reverse direction. (2) In a three point interallelic cross, the outermost alleles gave reciprocal recombination or conversion from mutant to wild on nine occasions in a sample of 1419 tetrads; whereas on the basis of the map distance between these sites about thirty-four rather than nine such events would have been expected. This suggests that in the three-point cross conversion and 
crossing-over is much rarer than in a two-point one. (3) One aberrant tetrad showed a 4:0 ratio for mutant 5, i.e. the mutant was not represented in the tetrad. On the copy choice model it must be assumed that the allele was lost by back mutation; yet on the basis of the reverse mutation rate which is given for this mutant, the chance of this event being detected in a sample of a few hundred tetrads would appear to be small. On the other hand if effective pairing occasionally involves all four rather than two chromatids then the opportunity for conversion of both mutant alleles in a tetrad would be present. At the cys region, the map based on prototroph frequencies among random spores shows two very distinct clusters of sites at the extreme ends of the map. Although within the clusters the linear order of sites is convincing there are distinct indications that map expansion may be present. Tetrad analysis of crosses between a mutant from each cluster has been carried out in such a way that only recombinant tetrads producing a wild-type spore pair would be detected. In sixty-seven such tetrads the wild-type spores were always generated by conversion of one or the other mutants in the cross. As has been explained, if conversion by repair at a given mispaired site can occur in either direction, then some wild-type spores should be formed in association with doubly defective ones, as occurs at the pan-2 locus. This would, however, be uncommon if most conversion occurred from mutant to wild allele. In crosses between mutant and wild alleles, there is indeed a strong indication of this asymmetry in conversion frequencies, although the authors believed that in their material conversion from mutant to wild would be detected more readily than the reverse situation. Alternatively, the failure to obtain doubly mutant spores could be explained on the basis of a modified conversion model which will be discussed in relation to the Ascobolus conversion data. As in the pan-2 analysis, there was no obvious polarity in the conversion event such has been demonstrated in Ascobolus; but amongst the convertant spores there does seem to be a distinct polarity in the behaviour of outside markers which is similar to that described by Murray (see below). A further point that emerges from these data is that there is no indication that conversion occurs at a higher rate in crosses between mutant and wild allele than in interallelic crosses. However, the very large empty region in the map between the clusters suggests that when intercrossed mutants are in different clusters their combined interference with pairing may be no greater than it would be in a one-point cross.

Several other series of mutants in Neurospora have been used in interallelic crosses in order to construct fine structure maps by random spore analysis, but extensive tetrad data is not available. The most detailed map is at the ad- 8 locus (Ishikawa, 1962). This is unusual in that no pronounced clustering of sites occurs; the linearity of sites and the additivity of recombination fractions is convincing, and no map expansion is evident. Is the lack of clustering correlated with the good linearity and additivity? At the other extreme ten am mutants when intercrossed in all pair-wise combinations are difficult to map in a convincing linear order (J. A. Pateman, personal communication). The data strongly suggest that the mutants themselves influence the amount of recombination, since there is a pronounced tendency for certain mutants to give high recombination with all the others, while 
other mutants behave in the reverse manner. Mutants at the nic-1 locus do not give a linear map either (St. Lawrence, 1956), unless map expansion is occurring; and mutants at the pyr-3 locus can be mapped in only an approximate linear order (Suyama, Munkres \& Woodward, 1959). In some of these studies the behaviour of outside markers has not been followed. That this may mean that much important information is missed is shown by the very detailed studies by Murray (1963) with mutants at the me-2 locus. These mutants fall into a linear map with distinct clusters of sites. The recombination frequencies are broadly additive, but different crosses between the same two mutants often gave markedly different frequencies of prototrophs. But her main result can best be explained by quoting from her own summary. 'Methionine independent progeny from crosses between many pairs of combinations involving alleles at the $m e-2$ locus were classified with respect to outside markers. One of the two classes of methionine prototrophs having outside markers recombined occurred in excess of the other. When the markers entered the cross in the opposite phase, a similar excess was found in the reciprocal class.' 'Pronounced asymmetries were also observed in the numbers of the two parentally marked classes; when the markers entered the cross in the opposite phase, these asymmetries were reversed. The asymmetries between the two parentally marked classes were correlated in direction with the asymmetries between the two classes having the markers recombined. The results may be interpreted in terms of multiple exchanges within small, discontinuously distributed, regions of effective pairing. The asymmetries could result from a reduction of co-incident exchanges in the region proximal to the selected interval, if the $m e-2$ region is situated immediately distal to some discontinuity in the genetic material.' These results are similar to although more extensive than those obtained in Aspergillus, and can be explained in the same way, if the discontinuity is distal to the me-2 region. Conversion of the distal allele in any cross with or without crossing-over between outside markers will produce an excess of one parental and one recombinant outside marker class. When the outside markers enter the cross in the opposite phase, then the other parental and other recombinant class are predominant. Some of the studies which have already been mentioned show similar patterns of outside marker behaviour, whereas others do not do so. This could be explained if there were sometimes discontinuities both proximal and distal to the region under examination, or perhaps within it.

\section{(iv) Sordaria fimicola}

Gene conversion in this species has been studied only in crosses between mutant and wild-type strains, but a number of revealing observations have been made (Kitani et al., 1961, 1962). (1) The spore colour mutant $g$ gives both 6:2 and 5:3 convertant asci, whereas the mutant $m$ gives only $6: 2$ asci. (2) The conversion from $g$ to the wild allele is very much more frequent than conversion in the reverse direction: the data from several crosses is given in Table 1. (3) Conversion is strongly correlated with recombination between outside markers-particularly with regard to the $5: 3$ asci-and only one pair of chromatids is usually involved in the two events. 
The observations are compatible with the repair model if it is supposed that for the $g$ mutant correction of mismatched bases sometimes does not take place before postmeiotic replication, but that such correction always occurs at the $m$ site; and that the correction at the $g$ site results in most cases in the establishment of the wildtype base pair configuration. In addition the several $4: 4$ aberrant asci which were also observed would result from asci where neither of the mispaired bases were corrected before post-meiotic replication. Special features would have to be added to the copy choice model in order to account for observations (1) and (2).

Table 1. Aberrant asci from crosses between the mutant $\mathrm{g}$ and its wild-type allele in Sordaria

$\begin{array}{cc}g:+ & \\ \text { ascus } & \text { Number } \\ \text { ratio } & \text { observed } \\ 2: 6 & 98 \\ 6: 2 & 13 \\ 3: 5 & 108 \\ 5: 3 & 20\end{array}$

(v) Saccharomyces cereviseae

Conversion has been consistently observed during standard genetic analysis in this organism. In general the rates of conversion are much higher than in other fungi, but there is considerable variation in frequency between different loci or between different alleles at one locus (Roman, 1963). The data are not yet sufficient to tell whether for any given mutant allele, conversion from mutant to wild differs in frequency from the reverse occurrence. Conversion has also been extensively studied in heteroallelic diploids-diploids which carry two mutations at different sites within one gene. Such diploids revert to wild-type following somatic nonreciprocal recombination (Roman, 1956), whereas at meiosis such recombination may be either reciprocal or non-reciprocal (Roman, 1958). Since ultra-violet light increases the rate of somatic conversion much more than it does somatic crossingover in the same diploid, it has been argued that two separate mechanisms are involved in these processes and only one of them is very sensitive to ultra-violet light (Roman \& Jacob, 1958). The repair model clearly suggests a basis for two mechanisms. It is possible that after ultra-violet light treatment, pairing and temporary annealing of DNA strands is induced, which would allow the opportunity for conversion, but that in the mitotic cell the subsequent events necessary for crossing-over are less commonly achieved. Indirect evidence for the induction of genetic pairing by ultra-violet light is discussed elsewhere (Holliday, 1961, 1964).

(vi) Ascobolus immersus

The most striking studies on gene conversion have been carried out by Lissouba, Rizet, Mousseau \& Rossignol. Their recent review (1962) of many extensive studies will be used as the basis for the discussion here. They have worked exclusively 
with spore colour mutants. These fall into several series where mutants within one series when intercrossed give very few wild-type spores and are presumed to be allelic. The main results are that within a series a group or groups of mutants can be found which when intercrossed give rare wild-type spores that arise only as a result of gene conversion; that the conversion frequencies can be used to map the sites in a linear order, and that the conversion is polarized such that in any cross only the mutant nearest to a specific end of the map shows conversion. At the end of this polaron, there is thought to be a discontinuity, linker or structure de liaison, the position of which is located by the fact that mutants spanning it show crossing-over or conversion, and the conversion can occur at either site. This result appears to be rather neatly explained by the copy choice hypothesis if it is supposed that genetic replication proceeds along the polaron towards the linker, but that occasionally one of the new chromatids switches and is copied off the other chromosome; at the linker a switch of either of the two new chromatids can occur. Thus from the point of the first switch to the linker there is a 3:1 segregation for any intervening site. In a cross between mutants, wild-type spores are of course produced only if the first switch occurs between the sites, and the chance of this happening depends on their distance apart. Unfortunately, there are a number of peculiarities in the overall data which cannot be easily reconciled with this hypothesis. (1) Mutants in series 75 could not on the whole be mapped in linear order owing to the non-additivity of recombination frequencies: a map for a small sample of the mutants is published but this shows only very rough linearity. The mutants of series 46 , which contains the most clear cut polaron, again show only approximate linearity and were it not for the polaron effect alternative orders would be almost as convincing. Two mutants within the polaron which have pink spores are not mapped and are reported to give abnormally high recombination with other mutants. (2) In series 75 some mutants when crossed produce asci showing post-meiotic segregation, others do not. (3) It has recently been reported (Rizet \& Rossignol, 1964) that when mutants are crossed with the wild-type, conversion of the wild allele to the mutant may not occur with the same frequency as conversion in the reverse direction. (4) In series 19 the mutants fall into three pronounced clusters A, B and C. This region exhibits map expansion with remarkable clarity. A number of crosses involving several mutants from each cluster gave homogeneous results which are shown in Table 2 (Table 3 in Lissouba et al., 1962). Crosses with other mutants in the series carried out at a different temperature gave heterogeneous results, but for any trio of markers, one in each of the clusters $\mathrm{A}, \mathrm{B}$ and $\mathrm{C}$, the $\mathrm{A} \times \mathrm{C}$ crosses always gave a much higher frequency of recombination than the sum of $\mathrm{A} \times \mathrm{B}$ and $\mathrm{B} \times \mathrm{C}$ (Table 4 in their paper). The polaron in series 46 also shows a cluster of mutants at one end, within which there are indications of map expansion. (5) It has been shown that the explanation of the polaron in terms of a copy choice is tenable only if certain inconsistencies in the data are disregarded (Fincham \& Day, 1963; Stadler, 1963). In addition, experiments in yeast and Neurospora which would have detected polarized conversion had it existed have given negative results.

Even if the polaron turns out to be a feature peculiar to Ascobolus, any alternative 
model to copy choice must be able to explain the main result of polarized conversion. Returning to the model proposed in this paper, it can be assumed that the polaron corresponds to the region to one side of the linker. In a cross between mutants in this region, the formation of hybrid DNA may often be terminated at the first point

Table 2. Summary of results obtained in crosses $A \times B, B \times C$ and $A \times C$ among mutants of Series 19 in Ascobolus

$\begin{array}{cccc} & & \text { Frequency of } \\ \text { Cross } & 8: 0 \text { asci } & 6: 2 \text { asci } & 6: 2 \text { asci } \\ \mathrm{A} \times \mathrm{C} & 85,170 & 133 & 1 \cdot 6 \pm 0.2 \times 10^{-3} \\ \mathrm{~B} \times \mathrm{C} & 45,000 & 71 & 1 \cdot 6 \pm 0.3 \times 10^{-3} \\ \mathrm{~A} \times \mathrm{C} & 26,440 & 495 & 19 \cdot 2 \pm 0.9 \times 10^{-3}\end{array}$

of heterozygosity-in which case there would be no opportunity for conversion. Occasionally, annealing may cross the first mutant site and either terminate between sites or get held up at the second site. Very rarely both sites may be crossed.

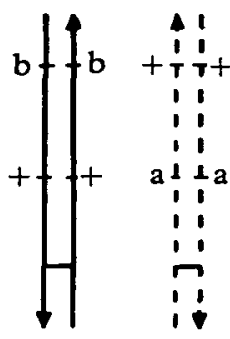

1

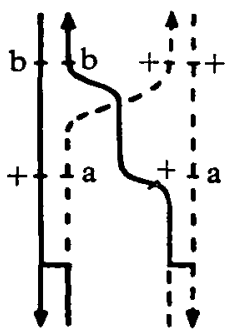

2

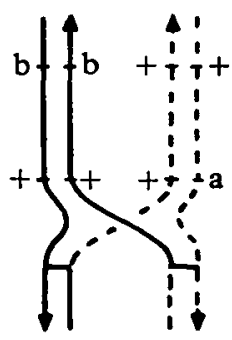

$3 a$

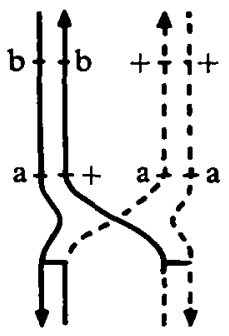

$3 b$

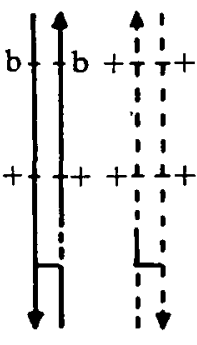

$4 a$

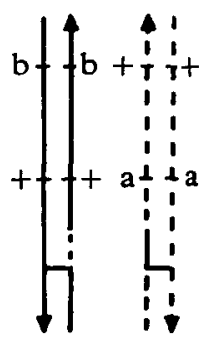

$4 \mathrm{~b}$

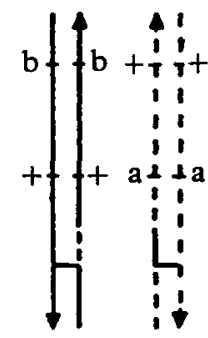

$4 \mathrm{c}$

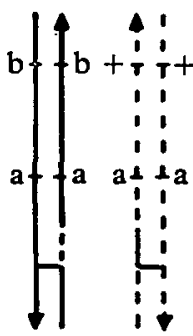

$4 \mathrm{~d}$

Fig. 5. An alternative configuration of the DNA to that shown in Fig. 1. $a$ and $b$ represent mutant alleles. The opportunity for conversion is restricted by the interference of heterozygous site $a$ with the formation of hybrid DNA. Initially only one mismatched pair is incorporated into double stranded DNA. Correction of this is followed by the formation of the half chromatid chiasma between $a$ and the linker, and leads to alternative secondary mismatching of bases in 3a or $3 \mathrm{~b}$. Each of these can be corrected in two ways to give the four possibilities in 4a-d without crossing over of chromatids, as drawn here, or with crossing over. In no case is a wild-type chromatid formed together with a doubly defective one. 
Thus conversion would nearly always affect the site nearest the linker; and mutants astride the linker would of course show crossing-over or conversion. Although this goes some way towards accounting for the polaron effect, it cannot be the complete explanation for the following reason. If the hybrid region spans the first site the two pairs of mis-matched bases might sometimes be corrected such that one mispair changes to the wild configuration and the other to the mutant. If this occurred at random, in half the cases a wild-type chromatid would be formed along with a doubly mutant one. This has been observed only once within a polaron. What seems to be required is a mechanism which results in both hybrid strands going either to the mutant or to the wild-type configuration. This could happen if strands tended to unravel and anneal more than once across the mutant site. Then in the presence of the active repair mechanism, the only stable condition would be that which is required to explain the data. (This possibility has already been discussed in relation to the induction of mutations in multistranded chromosomes (Holliday, 1962).) An alternative explanation which would also be compatible with the observations is shown in Fig. 5.

Only two of the four strands become annealed beyond the position of the first mutant site, and because of the heterozygosity this part of the annealed region is unstable. There would be the possibility of repair in either direction, but the strands would soon unravel to a position between the linker and the first mutant site. Breakage and reunion would occur here, and the newly annealed parental DNA would now contain mispaired bases and be repairable. The result would be that a doubly mutant strand would never be formed together with a wild-type one. If further studies of the cys region in Neurospora failed to reveal any tetrads of this type, then this modified model might also be applicable there and would appear to explain the behaviour of outside markers rather more easily than the model in Fig. 1.

\section{(vii) Other organisms}

It is not within the scope of this article to review the situation with regard to reciprocal and non-reciprocal recombination in organisms other than fungi, but a very brief discussion of some aspects of fine structure mapping in bacteriophage and bacteria may be of relevance to the general discussion. The $r$ II region of bacteriophage $\mathrm{T} 4$ has been more extensively mapped than any other (Benzer, 1961). It has been shown that two-point crosses between $r$ II mutants can be used to construct a map with an unequivocal linear order of sites, and within which there is no pronounced clustering of sites and no map expansion. In fact, the reverse is true: owing to the existence of negative interference, map distances within the region are always greater than recombination frequencies (Edgar, Feynman, Klein, Lielausis $\&$ Steinberg, 1962). Although sites are not clustered there are many sites, called hot spots, where a large number of individually isolated mutants map at the same position (Benzer, 1961). Tessman (1963) has recently presented evidence that these hot spots are in fact divisible by recombination. In other words a hot spot may be a cluster of sites. Furthermore, he shows that the recombination fractions between such sites are completely non-additive, which must indicate that the mutations 
affect the recombination at this level of analysis. The $r$ II region is several map units long; perhaps only very small parts of this are equivalent in terms of recombination to fine structure maps in fungi which can have a map length two orders of magnitude less than that of the $r \mathrm{II}$ region.

In fine structure analysis in bacteria by transduction, it has been consistently observed that some mutants give aberrant recombination frequencies (Demerec, Goldman \& Lahr, 1958; Hartman, Loper \& Šerman, 1960; Balbinder, 1962; Margolin, 1963), including isolated examples of the map expansion effect (Yanofsky \& Crawford, 1959). If these mutants are omitted additive linear maps can be constructed, but Smith (1961) was unable to map a group of MetB mutants in Salmonella. Again, in transformation experiments pronounced marker effects have been recorded (Ravin \& Iyer, 1962, and references therein). Since the general feeling is that any group of mutants should fall into a linear array if appropriate mapping techniques are available, it may well be that experiments with bacteria as well as fungi which have failed to achieve this result, have not been published. It is not possible to be certain whether all observed recombination in bacteriophage and bacteria is due to a single standard recombination mechanism, or whether events akin to the type of gene conversion discussed in this paper also operate. Experiments which are designed to provide definitive information about a particular aspect of the mechanism of recombination should not neglect this possibility. Thus two point crosses may yield an unknown proportion of conversion-type recombinants, whereas four-point crosses would yield information that made it much more certain when standard recombination was being observed.

\section{TESTING THE MODEL}

Evidence in favour of the proposed mechanism for gene conversion might be obtained by the following means:

(1) If it could be shown that conversion and crossing-over could occur in the absence of any DNA synthesis, all copy choice models would be ruled out, and the mechanism proposed here would be a reasonable alternative. So far there is no evidence from fungi relevant to this point; but experiments which might provide some are feasible.

(2) In many cases a cross between a revertible auxotroph and its wild-type allele, yields aberrant tetrads containing three auxotrophic and one wild-type spore. According to the repair model two of these auxotrophs should have arisen by the correction of mispaired bases in the region of hybrid DNA. The subsequent paired bases need not be the same as they were in the original parental auxotroph, although they may give the same or similar mutant phenotype. If this were so, then the reverse mutation rate of the three auxotrophs might be found to be heterogeneous, since different mutant base pairs would not be expected to change by spontaneous mutation to the wild-type with equal frequency. Or, differential response of the auxotrophs to specific mutagens might be detected.

(3) A similar test to (2) could be made by using a gene which controls the structure of a known enzyme. Here, in a cross between a mutant lacking enzyme activity and 
wild-type, the aberrant tetrads with three phenotypically wild-type spores could be used to determine whether the enzyme from these three progeny was identical in properties or not. It might sometimes be found that the convertants had reduced enzyme activity or an enzyme with distinct kinetic behaviour. In the same way, recombinants from crosses between different mutant alleles might produce functional enzyme which was distinguishable from wild-type.

(4) The effect of heterozygosity on recombination could be tested in organisms such as Drosophila or Neurospora where groups of very closely linked markers are available. If these markers were all heterozygous in a cross, then the recombination between the outermost markers might be less than in a cross where the intervening region was homozygous.

If experiments (2) or (3) gave positive results, this would provide very strong evidence for conversion by repair. However, a negative result would not be evidence against the hypothesis. Since if only one of the mispaired bases in the hybrid region was replaced, then the consequent normal base pair would always be the same as one or other of the parents. Only if both of the mispaired bases were changed could one of the other two possible base pairs arise at that position, and in this case genetically distinct new convertant alleles might be identified.

Preliminary experiments have been carried out with yeast which do indeed suggest a positive result for experiment (2). In aberrant tetrads containing three copies of an auxotrophic mutant, reversion rates varied by a factor of thirty; whereas when mutant strains from a number of normal tetrads were examined there was considerably less variation in reversion rate. These results will be reported in detail elsewhere. On the other hand, results reported by Giles (1958) and Case \& Giles (1963) with Neurospora indicate negative results for tests (2) and (3).

\section{SUMMARY AND CONCLUSION}

A mechanism for gene conversion is proposed which overcomes many of the difficulties that any copy choice model encounters. It is suggested that along with general genetic pairing of homologous genomes at meiosis, effective pairing over short regions of the genetic material occurs at the molecular level by the separation of the strands of the DNA double helices, followed by the annealing of strands from two homologous chromatids. If the annealed region happens to span a heterozygous site, mispairing of bases will occur. Such a situation may be analogous to that in DNA which is damaged by mutagens; the same or similar repair mechanisms may operate, and these, by adjusting the base sequences in order to restore normal base pairing, would bring about gene conversion in the absence of any genetic replication. The model indicates how precise breakage and rejoining of chromatids could occur in the vicinity of the conversion, so that conversion would frequently be accompanied by the recombination of outside markers. The model also proposes that the distance between two mutant sites on a fine structure map depends not so much on the frequency of a recombinational event occurring between them, but rather on the degree of inhibition of the processes of genetic pairing by the mutants themselves.

The model will explain almost all the data in a formal way, and it has the advant- 
age over copy choice mechanisms for gene conversion in (1) being compatible with semi-conservative replication of DNA, (2) not invoking DNA synthesis during or after genetic pairing, (3) providing a molecular mechanism for close specific pairing, (4) making it unnecessary to postulate sister strand exchange or a process akin to this, (5) suggesting why rates of gene conversion in opposite directions are sometimes unequal and (6) providing an explanation of the clustering of mutant sites, a basis for map expansion and for the apparently capricious departure of fine structure maps from additivity. Although the model proposed is a general rather than a specific one, it suggests that the process of conversion and intragenic recombination is more complex than is usually believed, since it depends on several interacting factors. Nevertheless, it is hoped that the introduction of a model with this complexity will help to stimulate specific experiments, and that these will provide definitive information which would never be obtained if simpler models of conversion and intragenic recombination were believed to explain the genetic data sufficiently well.

I am particularly grateful to Drs U. Leupold and H. Gutz for making available to me the extensive unpublished data that were used in Fig. 2, and also to Drs J. A. Pateman and J. J. Weigle for allowing me to refer to some of their unpublished results. I thank Drs L. Sandler and J. R. S. Fincham for their encouragement and helpful discussions.

\section{REFERENCES}

Batbinder, E. (1962). The fine structure of the loci try $\mathrm{C}$ and try $\mathrm{D}$ of Salmonella typhimurium. II. Studies of reversion patterns and the behaviour of specific alleles during recombination. Genetics, 47, 545-559.

Benzer, S. (1961). On the topography of the genetic fine structure. Proc. nat. Acad. Sci., Wash., 47, 403-415.

CASE, M. E. \& GiLes, N. H. (1958). Recombination mechanisms at the pan-2 locus in Neurospora crassa. Cold Spr. Harb. Symp. quant. Biol. 23, 119-135.

CASE, M. E. \& GrLes, N. H. (1963). In Methodology in Basic Genetics (W. J. Burdette, ed.). Pp. 221-226. San Francisco: Holden-Day Inc.

Demerec, M., Goldman, I. \& LAHR, E. L. (1958). Genetic recombination by transduction in Salmonella. Cold Spr. Harb. Symp. quant. Biol. 23, 59-68.

Edgar, R. S., Feynman, R. P., Klein, S., Lielausis, I. \& Steinberg, C. M. (1962). Mapping experiments with $r$ mutants of bacteriophate T4D. Genetics, 47, 179-186.

Fincham, J. R. S. \& Day, P. R. (1963). Fungal Genetics. Oxford: Blackwell.

Fresco, J. R. \& Alberts, B. M. (1960). The accomodation of noncomplementary bases in helical polyribonucleotides and deoxyribonucleic acids. Proc. nat. Acad. Sci., Wash., 46, $311-321$.

Giles, N. H. (1958). Mutations at specific loci in Neurospora. Proc. X Int. Congr. Genet. 1, 261-279.

Gutz, H. (1961). Distribution of X-ray and nitrous acid-induced mutations in the genetic fine structure of the ad-7 locus of Schizosaccharomyces pombe. Nature, Lond., 191, 1125-1126.

Hartman, P. E., Loper, J. C. \& Serman, D. (1960). Fine structure mapping by complete transduction between histidine requiring Salmonella mutants. J.gen. Microbiol. 22, 323-353.

HersheY, A. D. (1958). The production of recombinants in phage crosses. Cold Spr. Harb. Symp. quant. Biol. 23, 19-46.

Hexter, W. M. (1963). Non-reciprocal events at the garnet locus in Drosphila melanogaster. Proc. nat. Acad. Sci., Wash., 50, 372-379.

Holitid Ay, R. (1961). Induced mitotic crossing-over in Ustilago maydis. Genet. Res. 2, 231-248.

Holliday, R. (1962). Mutation and replication in Ustilago maydis. Genet. Res. 3, 472-486. 
Holliday, R. (1964). The induction of mitotic recombination by mitomycin C in Ustilago and Saccharomyces. Genetics (in press).

Ishikawa, T. (1962). Genetic studies of ad-8 mutants in Neurospora crassa. I. Genetic fine structure of the ad 8 locus. Genetics, 47, 1147-1161.

KellenbURGer, G., ZICHCHI, M. L. \& Weigle, J. J. (1961). Exchange of DNA in the recombination of bacteriophage. Proc. nat. Acad. Sci., Wash. 47, 869-878.

Kitani, Y., OLIvE, L. S. \& EL-ANI, A. S. (1961). Transreplication and crossing-over in Sordaria fimicola. Science, 134, 668-669.

Krtani, Y., Olrve, L. S. \& El-AnI, A. S. (1962). Genetics of Sordaria fimicola. V. Aberrant segregation at the $g$ locus. Amer.J. Bot. 49, 697-706.

LEUPOLD, U. (1961). Intragene Rekombination und allele Komplementierung. Arch. KlausStift. VererbForsch. 36, 89-117.

LINDEGREN, C. C. (1953). Gene conversion in Saccharomyces. J. Genet. 51, 625-637.

Lissouba, P., Moussead, J., Rizet, G. \& Rossignol, J. L. (1962). Fine structure of genes in the Ascomycete Ascobolus immersus. Advanc. Genet. 11, 343-380.

Margolin, P. (1963). Genetic fine structure of the leucine operon in Salmonella. Genetics, 48, 441-457.

Meselson, M. \& Weigle, J. J. (1961). Chromosome breakage accompanying genetic recombination in bacteriophage. Proc. nat. Acad. Sci., Wash., 47, 857-868.

Mitchell, M. B. (1955). Aberrant recombination of pyridoxine mutants of Neurospora. Proc. nat. Acad. Sci., Wash., 41, 215-220.

Murray, N. E. (1963). Polarized recombination and fine structure within the me-2 gene of Neurospora crassa. Genetics, 48, $1163-1183$.

Nesson, O. E. (1962). The waxy locus in maize, I. Intra locus recombination frequency estimates by pollen and by conventional analyses. Genetics, 47, 737-742.

Perkins, D. D. (1962). The frequency in Neurospora tetrads of multiple exchanges within short intervals. Genet. Res. 3, 315-327.

Ponteconvo, G. (1958). Trends in Genetic Analysis. New York: Colombia University Press.

Pritchard, R. H. (1955). The linear arrangement of a series of alleles of Aspergillus nidulans. Heredity, 9, 343-371.

Pritchard, R. H. (1960a). The bearing of recombination analysis at high resolution on genetic fine structure in Aspergillus nidulans and the mechanism of recombination in higher organisms. Symp. Soc. gen. Microbiol. 10, 155-180.

Pritchard, R. H. (1960b). Localized negative interference and its bearing on models of gene recombination. Genet. Res. 1, 1-24.

Putrament, A. (1963). Mitotic recombination within the paba-1 region of Aspergillus nidulans. (Abstr.). Proc. 11th Int. Congr. Genet. 1, 14.

RAVIN, A. W. \& IYer, V. N. (1962). Genetic mapping of DNA: influence of the mutated configuration on the frequency of recombination along the length of the molecule. Genetics, 47, $1369-1384$.

Rizet, G. \& Rossignol, J. L. (1964). Recombination within one locus of Ascobolus immersus. (Abstr.) Proc. XI Int. Congr. Genet. 2, (in press).

RomaN, H. L. (1956). Studies of genemutation in Saccharomyces. Cold.Spr. Harb.Symp.quant. Biol. 21, 175-183.

Roman, H. L. (1958). Sur les recombinaisons non reciproques chez Saccharomyces cereviseae et sur les problèmes posés par ces phenomènes. Ann. Genet. 1, 11-17.

Romas, H. L. (1963). Genic conversion in fungi. In Methodology in Basic Genetics (W. J. Burdette, ed.). Pp. 209-221. San Francisco: Holden-Day Inc.

Roman, H. L. \& JACOB, F. (1958). A comparison of spontaneous and ultraviolet-induced allelic recombination with reference to the recombination of outside markers. Cold Spr. Harb. Symp. quant. Biol. 23, 155-160.

St. Lawrence, P. (1956). The $q$ locus of Neurospora crassa. Proc. nat. Acad. Sci., Wash., 42 , 189-194.

SIDDIQ1, O. H. (1962). The fine genetic structure of the paba1 region of Aspergillus nidulans. Genet. Res. 3, 69-89.

SIDDIQI, O. H. (1963). The incorporation of parental DNA into genetic recombinants of $E$. coli. Proc. nat. Acad.Sci., Wash., 49. 589-592. 
Siddiqi, O. H. \& PUtrament, A. (1963). Polarized negative interference in the paba1 region of Aspergillus nidulans. Genet. Res. 4, 12-20.

SmrtH, D. A. (1961). Some aspects of the genetics of methionineless mutants of Salmonella typhimurium. J.gen. Microbiol. 24, 335-353.

SobeLs, F. H. (ed.). (1963). Repair from Genetic Radiation Damage. Oxford: Pergamon Press.

StadLER, D.R. (1963). Observations on the polaron model for genetic recombination. Heredity, $18,233-242$.

Stadler, D. R. \& Towe, A. M. (1963). Recombination of allelic cysteine mutants in Neurospora. Genetica, 48, 1323-1344.

Suyama, J., Munkres, K. D. \& Woodward, V. W. (1959). Genetic analysis of the pyr-3 locus of Neurospora crassa; the bearing of recombination and gene conversion upon intra-allelic linearity. Genetica, 30, 293-311.

Tessman, I. (1963). Genetic ultrafine structure of the T4r II region. (Abstr.). Proc. XI Int. Congr. Genet. 1, 10.

Whitehouse, H. L. K. (1963). A theory of crossing-over by means of hybrid deoxyribonucleic acid. Nature, Lond., 199, 1034-1040.

YANOFSKY, C. \& CRAWFORd, I. P. (1959). The effects of deletions, point mutations, reversions and suppressor mutations on the two components of the tryptophan synthetase of Escherichia coli. Proc. nat. Acad. Sci., Wash., 45, 1016-1026. 